\title{
NAR: Algoritmo de Roteamento Assistido para Comunicação D2D em Redes Celulares de 5G
}

\author{
Alex Vidigal Bastos ${ }^{1,2}$, Cristiano M. Silva ${ }^{1}$, Diógenes Cecílio da Silva Júnior ${ }^{2}$ \\ ${ }^{1}$ DTECH - Departamento de Tecnologia \\ Universidade Federal de São João del-Rei (UFSJ) - Ouro Branco, MG - Brasil \\ ${ }^{2}$ Programa de Pós-Graduação em Engenharia Elétrica \\ Universidade Federal de Minas Gerais - UFMG \\ Av. Antônio Carlos 6627, 31270-901, Belo Horizonte, MG - Brasil \\ \{alexvbh, cristiano\}@ufsj.edu.br, diogenes@ufmg.br
}

\begin{abstract}
This paper presents a routing algorithm for device-to-device (D2D) communication in cellular architectures with the goal to extend the coverage of base stations. The proposed algorithm takes into account that D2D communications in cellular networks are managed by base stations and allocation of resource blocks is formulated as a Minimum Graph Coloring Problem. The Load Balancing Based Selective Ad Hoc On-Demand Multipath Distance Vector (LBS-AOMDV) algorithm is considered as baseline. Experiments taking place on SimuLTE (OMnet++) reveal that the NAR algorithm has an energetic gain of approximately $16 \%$ compared to $L B S-A O M D V$, while sending approximately $51 \%$ more messages compared to LBS-AOMDV.
\end{abstract}

Resumo. Este artigo apresenta um algoritmo de roteamento para comunicação dispositivo a dispositivo (D2D) em arquiteturas celulares com o objetivo de ampliar a cobertura das estações base. O algoritmo proposto leva em consideração que as comunicações $\mathrm{D} 2 \mathrm{D}$ em redes celulares são gerenciadas por estações base e a alocação de blocos de recursos é formulada como um problema mínimo de coloração de grafos. O algoritmo Load Balancing Based Selective Ad Hoc On-Demand Multipath Distance Vector (LBS-AOMDV) é considerado como baseline. Os experimentos foram realizados no SimuLTE (OMnet ++) e demonstram que o NAR tem um ganho energético de aproximadamente $16 \%$ comparado ao LBS-AOMDV, enquanto envia aproximadamente $51 \%$ a mais de mensagens em relação ao LBS-AOMDV.

\section{Introdução}

A quinta geração de sistemas móveis corresponde a uma rede heterogênea, permitindo uma maior densidade de usuários de banda larga móvel, bandas de espectro múltiplo e múltiplos tipos de dispositivos suportando comunicações dispositivo a dispositivo (D2D) [Agiwal et al. 2016]. A comunicação dispositivo a dispositivo (D2D) é uma tecnologia candidata para ampliar a cobertura fornecida pelas estações base. Pesquisadores estão discutindo os prós e contras da aplicação da tecnologia D2D na arquitetura Long Term Evolution Advanced (LTE-A) [Lin et al. 2014]. De fato, é fundamental repensar as arquiteturas de comunicação móvel para reduzir os custos de manutenção, proporcionando flexibilidade para a implantação de serviços inovadores [Agiwal et al. 2016]. 
Nesse trabalho, apresentamos o NAR (algoritmo de roteamento assistido por rede), projetado para auxiliar na ampliação da cobertura celular através da comunicação D2D e fornecer comunicação D2D em situações de emergência dado que um conjunto de estações base se tornam indisponíveis devido a eventos não planejados.

Diferentemente de outras abordagens, o algoritmo proposto considera a alocação de blocos de recursos com base no problema mínimo de coloração de grafos. Como premissa, o algoritmo precisa de pelo menos uma estação base ativa para fornecer um melhor gerenciamento da alocação de recursos, diminuição da sobrecarga da rede celular, melhor gerência de interferências e participação da estação base.

Consideramos como baseline o algoritmo Load Balancing Based Selective Ad hoc On-Demand Multipath Distance Vector (LBS-AOMDV) proposto em [Tata and Kadoch 2014]. Ambos os algoritmos são implementados no SimuLTE [Virdis et al. 2014] (baseado em OMnet ++) e os resultados demonstram que o NAR tem um ganho energético de aproximadamente $16 \%$ comparado ao LBS-AOMDV, enquanto envia aproximadamente $51 \%$ a mais de mensagens em relação ao LBS-AOMDV.

Esse trabalho está organizado da seguinte forma: a Seção 2 apresenta os trabalhos relacionados. A Seção 3 apresenta o algoritmo NAR. A Seção 4 apresenta o algoritmo usado como baseline. A Seção 5 apresenta a metodologia. A Seção 6 compara ambas as abordagens. A Seção 7 conclui o documento.

\section{Trabalhos Relacionados}

A comunicação D2D está atraindo a atenção da indústria e da academia. Apresentamos nesta seção, trabalhos abordando aspectos relacionados à interferência, comunicação de múltiplos saltos, estudos teóricos, gerenciamento de desempenho e a aplicação da teoria dos jogos para resolver aspectos da comunicação D2D. Os autores de [Asadi et al. 2014] categorizam os vários tipos de comunicações D2D agrupadas em comunicação dentro da banda e fora da banda. A comunicação dentro da banda compartilha o espectro entre a comunicação D2D e as comunicações celulares. Por outro lado, a comunicação fora da banda explora conexões fora do espectro celular, a fim de reduzir o risco de interferência.

A interferência é uma questão importante ao se considerar as comunicações D2D. [Sharma and Roberts 2015] projetam um algoritmo eficiente para equilibrar a carga entre diferentes níveis das células, permitindo que mais usuários acessem recursos simultaneamente. [Yuan et al. 2014] apresentam o Interference Aware Routing (IAR) para reduzir a interferência D2D causada pela estação base. O roteamento ocorre ao longo da borda da célula da estação base. Quando a distância entre o dispositivo móvel e a estação base é maximizada, a interferência agregada é minimizada. Em [Lee and Chung 2013] observam que a interferência entre as entidades de comunicação celular e D2D podem ocorrer enquanto compartilha o mesmo recurso. Eles propõem um esquema de alocação de recursos para reduzir possíveis interferências entre os dispositivos.

A comunicação de múltiplos saltos também é explorada. [Melki et al. 2016] propõem uma alocação de recursos de rádio para rotas $\mathrm{D} 2 \mathrm{D}$ de múltiplos saltos com base em uma abordagem de prevenção de interferência. Em [Jingyi et al. 2015], os autores fornecem um algoritmo de roteamento de múltiplos saltos com eficiência energética analisado para um sistema D2D de múltiplos saltos. [Laha et al. 2015] propõem 
o conceito de redes de smartphones de múltiplos saltos baseado na tecnologia WiFiDirect e propõem um protocolo de roteamento baseado em cluster de energia eficiente (QGRP) para resolver o problema de energia devido ao gasto energético dos smartphones. [Nunes and Loureiro 2016] propõem um protocolo probabilístico de roteamento oportunístico que considera reuniões de grupos. O algoritmo proposto em relação ao estado da arte conseguiu aproximadamente a mesma taxa de entrega com redução de $40 \%$ na sobrecarga da rede.

Também encontram-se estudos teóricos e analíticos sobre a comunicação D2D. [Jiang et al. 2015] fornecem uma análise teórica e demonstram que o objetivo de maximizar a descarga celular é equivalente a maximizar o bem-estar social em uma rede comercial em que o conteúdo a ser compartilhado é a mercadoria e os usuários móveis são compradores ou vendedores. [Wang et al. 2015] apresentam uma estrutura teórica para analisar o desempenho das comunicações D2D na existência de interferência co-canal de outras transmissões D2D e celulares. Os autores consideram a comunicação D2D de múltiplos saltos usando o algoritmo de roteamento de caminho mais curto [Yuan et al. 2014] nos canais de ligação uplink e de ligação downlink. [Sedidi and Kumar 2016] propõem protocolos de troca de chaves para comunicação D2D. Os protocolos propostos são baseados na troca de chaves baseada no padrão Diffie-Hellman (DH) e funções criptográficas.

A literatura também apresenta estudos abordando o desempenho das redes D2D. Felice e Bononi [Felice and Bononi 2016] fornecem os resultados experimentais da tecnologia Wi-Fi Direct em um banco de provas composto por múltiplos smartphones heterogêneos. Os autores analisam os principais fatores que afetam o desempenho do sistema e investigam como criar redes de múltiplos grupos ponto a ponto (P2P), alavancando a presença de dispositivos de retransmissão P2P que são responsáveis pela descarga de dados entre diferentes grupos P2P. Em [Nardini and Stea 2016], os autores apresentam uma estrutura para selecionar quais comunicações devem usar o modo D2D e quando, e os recursos de destino para usuários D2D e não D2D, explorando a reutilização para o primeiro. Os dois problemas são demonstrados, embora aparentemente similares, devem ser mantidos separados e resolvidos em diferentes prazos, a fim de evitar problemas como a perda excessiva de pacotes. Foram modelados como problemas de otimização e proposto uma solução heurística para o segundo problema.

A teoria dos jogos também é aplicada em alguns trabalhos. [Zhu et al. 2015] fornecem um problema de incentivo nas comunicações D2D, modelando o incentivo nos cenários de descarregamento como um jogo. Uma rede comercial é construída entre estações base e usuários, em que os leilões são conduzidos para agrupar usuários e determinar recompensas adequadas.

Por fim, [Tata and Kadoch 2014] apresentam o Load Balancing Based Selective Ad Hoc On-Demand Multipath Distance Vector (LBS-AOMDV), um protocolo de roteamento usado para calcular múltiplas rotas entre dois pontos finais. LBS-AOMDV é extensivamente discutido na Seção 4.

Este trabalho se difere dos anteriores ao propor um algoritmo de roteamento que usa a estação base para gerenciar e definir as rotas a serem usadas na comunicação D2D, analisando o caminho mais curto e a qualidade do canal, alocando recursos para as rotas selecionadas através de um problema de coloração de grafos. 


\section{NAR: Algoritmo de Roteamento Assistido pela Rede}

NAR (Network Assisted Routing) [Bastos and Silva 2018] é um algoritmo de roteamento dentro da banda que visa uma cobertura temporária quando as estações base enfrentam falhas técnicas. As características mais importantes do NAR são: a) o roteamento D2D é definido pelas estações base; b) a comunicação D2D ocorre no espectro celular (dentro da banda) e c) cada rota possui recursos correspondentes alocados para a comunicação D2D.

NAR define os múltiplos caminhos entre a estação base e os dispositivos móveis usando o caminho mais curto e o caminho com melhor qualidade do canal (CQI) dentro de um limite parametrizado, permitindo que as estações base desempenhem um papel importante ao gerenciar as rotas entre os dispositivos móveis dentro da célula. Para o funcionamento do algoritmo NAR, a rede é modelada como um grafo onde cada nó corresponde a uma comunicação D2D, sendo uma cor atribuída a cada canal não adjacente, e a alocação de blocos de recursos realizada através do problema de coloração de grafos.

\subsection{Modelo da Rede}

Nesta Seção, é formulado o problema de alocação de blocos de recursos para a comunicação D2D. Em primeiro lugar, descrevemos o modelo de comunicação D2D e formalizamos o problema baseado em uma abordagem de coloração de grafo.

Cada bloco de recurso (RB) denotado por $\beta_{i}$ é associado com uma transmissão de um dispositivo móvel $U E_{i}$ para a $U E_{j}$, baseado na qualidade do canal (CQI) de comunicação, em que o CQI define o RB utilizado $\beta_{i}^{1}, \beta_{i}^{2}, \beta_{i}^{3}, \beta_{i}^{4}$. O objetivo é minimizar as interferências entre os canais de comunicação de forma a maximizar a qualidade do canal. Modela-se o conjunto $B$ abrangendo as comunicações D2D em andamento e as novas solicitações de comunicação de entrada (ex: $B=b_{i}^{j}$ ), como um grafo não direcionado $G=(V, E)$. Cada $v \in V$ corresponde a uma comunicação $b_{i}^{j}$ do $U E_{i}$ para a $U E_{j}$. Obviamente, para cada comunicação $b_{i}^{j}, U E_{i}$ deve estar localizado dentro das faixas de frequências determinadas $T_{R}$ de $U E_{i}$.

Uma aresta $\epsilon=(n 1, n 2) \in E$ existe somente se $n 1$ é capaz de interferir com $n 2$ e vice-versa. A interferência é modelada entre duas comunicações D2D $b_{i}^{j}$ e $b_{k}^{l}$ da seguinte forma: a) transmissor $U E_{i}$ interfere com o receptor $U E_{l}$ ou b) o transmissor $U E_{k}$ interfere com o receptor $U E_{j}$. De fato, a interferência ocorre se: a) a distância de separação entre $U E_{i}$ e $U E_{l}$ ou entre $U E_{k}$ e $U E_{j}$ for inferior ao intervalo de interferências $I_{R}$ e b) os transmissores enviarem os quadros simultaneamente.

A avaliação da interferência é fundamental para melhorar os requisitos como: atraso, taxa de transferência e latência dentro da comunicação D2D. Neste trabalho, é utilizado o modelo de transmissão de sinais de Friis [Shaw 2012]. A utilização deste modelo é motivada pelo fato de que não existem obstáculos no ambiente. Cada transmissor $(U E)$ é caracterizado por um conjunto de parâmetros expressos em $d B$ : a) potência de transmissão $P_{t}$, b) recepção da potência do sinal $P_{r}$, c) ganho da antena transmissora $G_{t}$ e d) ganho da antena de recepção $G_{r}$. Baseado no modelo de Friis, a força do sinal de recepção $P_{r}$ é expressa na Eq. 1 como:

$$
P_{r}=P_{t}+G_{r}+G_{t}+20 \log 10\left(\begin{array}{c}
\lambda \\
4 \pi . d
\end{array}\right)^{\alpha} \ldots d B
$$


onde $\lambda$ é o comprimento de onda, $d$ é a distância entre os $U E s$ em metros e $\alpha$ é o modelo de perda do caminho.

O modelo de Sinal de Interferência de Rádio (SINR) adotado é descrito na Eq. 2. Seja $P_{r}\left(U E_{i}, U E_{j}\right)$ a intensidade do sinal de $U E_{i}$ recebido em $U E_{j}$. Um sinal transmitido a partir de $U E_{i}$ é realizado com sucesso em $U E_{j}$ se a) $P_{r}\left(U E_{i}, U E_{j} \geq R_{x_{-}} T h r\right)$ em que $R_{x \_} T h r$ é um limiar predefinido que corresponde a sensibilidade do $U E$ e b) a relação entre $P_{r}\left(U E_{i}, U E_{j}\right)$ e a interferência total recebida em $U E_{j}$ do restante dos $U E s$ e $k \neq$ $i$ na rede excedendo um determinado limiar $C P_{-} T h r$, sendo $P_{I}\left(U E_{k}, U E_{j}\right)$ indica a potência de interferência recebida em $U E_{j}$ causada por $U E_{K}$.

$$
\begin{array}{r}
\operatorname{SINR}\left(U E_{i}, U E_{j}\right)=\frac{P_{r}\left(U E_{i}, U E_{j}\right)}{\sum_{U E_{k} \neq U E_{i}} P_{I}\left(U E_{k}, U E_{j}\right)} \\
\geq C P \_T h r
\end{array}
$$

O objetivo é maximizar a proporção de $b_{i}^{j}$ para a comunicação D2D e minimizar a interferência na rede. Para fazer isso, a alocação de blocos de recursos para a comunicação D2D deve ser otimizada em que a decisão é tomada na chegada de cada nova requisição. Para alcançar o objetivo, os blocos de recursos são dinamicamente atribuídos as comunicações D2D. Além disso, devido ao número limitado de blocos de recursos, essa característica se torna essencial para o modelo.

A formulação do problema de alocação de blocos de recursos é definida como um problema de coloração de grafo mínimo (Min-GP) [Mehrotra and Trick 1995] de tal forma que cada nó $n \in V$ terá exatamente uma cor, garantindo que dois nós adjacentes têm diferentes cores, tendo como objetivo minimizar o número de cores usadas para cobrir todos os nós do grafo.

Denota-se $P$ o conjunto de todos os conjuntos estáveis máximos em $G$. Lembra-se que um conjunto estável é um subconjunto de $V$ que é composto por nós não adjacentes correlacionados. Um conjunto estável máximo é um conjunto estável que não é estritamente incluído em qualquer outro conjunto estável. Vale ressaltar que todos os nós no conjunto estável podem ser atribuídos uma cor, uma vez que eles não são vizinhos. Dessa forma, o grupo de comunicações D2D correspondente aos nós no conjunto estável faz uso do mesmo bloco de recurso.

Nosso objetivo é calcular o número mínimo de conjuntos estáveis, em que $k$ abrange todos os nós em $G$. Tal número corresponde a $k$-coloração e é denominado de número cromático de $G$, denotado por $\chi(G)$. Para calcular $\chi(G)$, nosso problema é formulado como programação inteira com base na formulação de conjunto independente.

$$
\begin{array}{r}
\chi(G)=\min \sum H \in P^{x_{H}} \\
\text { sujeito } a: \forall n \in V, \sum H \in P\left(x_{H} \cdot 1_{\{n \in H\}}\right) \geq 1 \\
\forall H \in P, x_{H} \in\{0,1\}
\end{array}
$$

Na Eq. 3, o problema de alocação de recursos é definido como: a) $x_{H}$ é uma variável binária que define se $H \in P$ é atribuída uma cor ou não e b) $1\{n \in H\}$ é uma função de indicação, isto é igual a 1 se a condição $n \in P$ é verdade, caso contrário, é 0 . De acordo com a restrição, cada nó $n \in V$ deve ter pelo menos uma cor. Assim, a ideia é selecionar uma cor entre as atribuídas a $n$. 


\subsection{O Algoritmo NAR}

O diagrama de sequência exibido na figura 1 descreve como os dispositivos móveis (UEs) e a estação base (eNB) trocam mensagens ao longo do tempo, descrevendo a interação entre os objetos (estação móvel, dispositivo móvel da borda e dispositivos móveis).

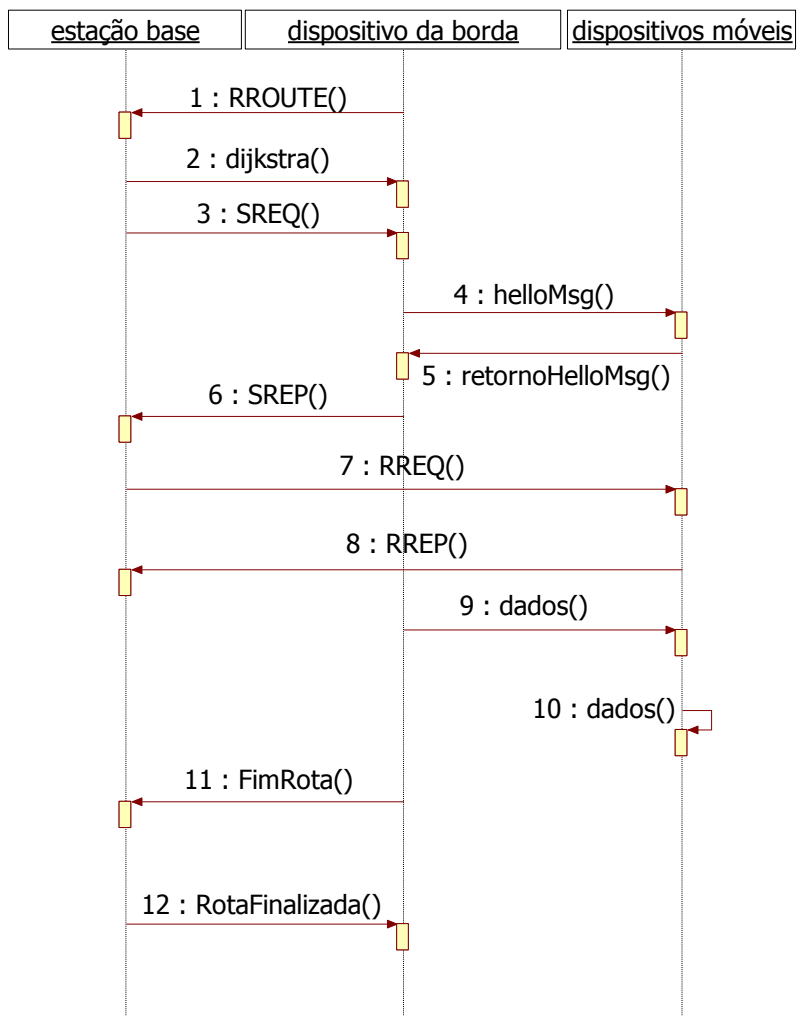

Figura 1. Diagrama de Sequência do NAR.

NAR é formalmente apresentado no Algoritmo 1. Ele começa quando a eNB recebe uma solicitação (RROUTE) de um UE da borda para executar uma comunicação D2D. O algoritmo Dijkstra() é executado para encontrar o menor caminho entre a eNB e o dispositivo móvel da borda. Um pacote de solicitação $(S R E Q)$ é enviado da eNB para o UE da borda solicitando a qualidade do canal de cada vizinho do dispositivo móvel. Antes de enviar a resposta $(S R E P)$, o UE da borda transmite uma mensagem Hello (Hello - msg) com um tempo de vida (TTL) igual a 1 para todos os seus vizinhos. Cada vizinho que recebe a mensagem retorna a qualidade do canal (CQI). O conjunto de vizinhos e os CQIs são transmitidos para a eNB através de um pacote de resposta $S R E P$ [Bastos and Silva 2017].

Os atributos $R R E Q_{T X}$ e $R R E Q_{R X}$ são utilizados para armazenar os valores de ativação (ligado) e desativação (desligado) que determinam se o dispositivo móvel já recebeu a mensagem de rota. Para garantir o loop-free das múltiplas rotas, cada dispositivo envia um $R R E Q$ para definir o atributo $R R E Q_{T X}$ como desativado. Além disso, cada dispositivo móvel que recebe o pacote deve desligar o atributo $R R E Q_{R X}$. Uma exce- 


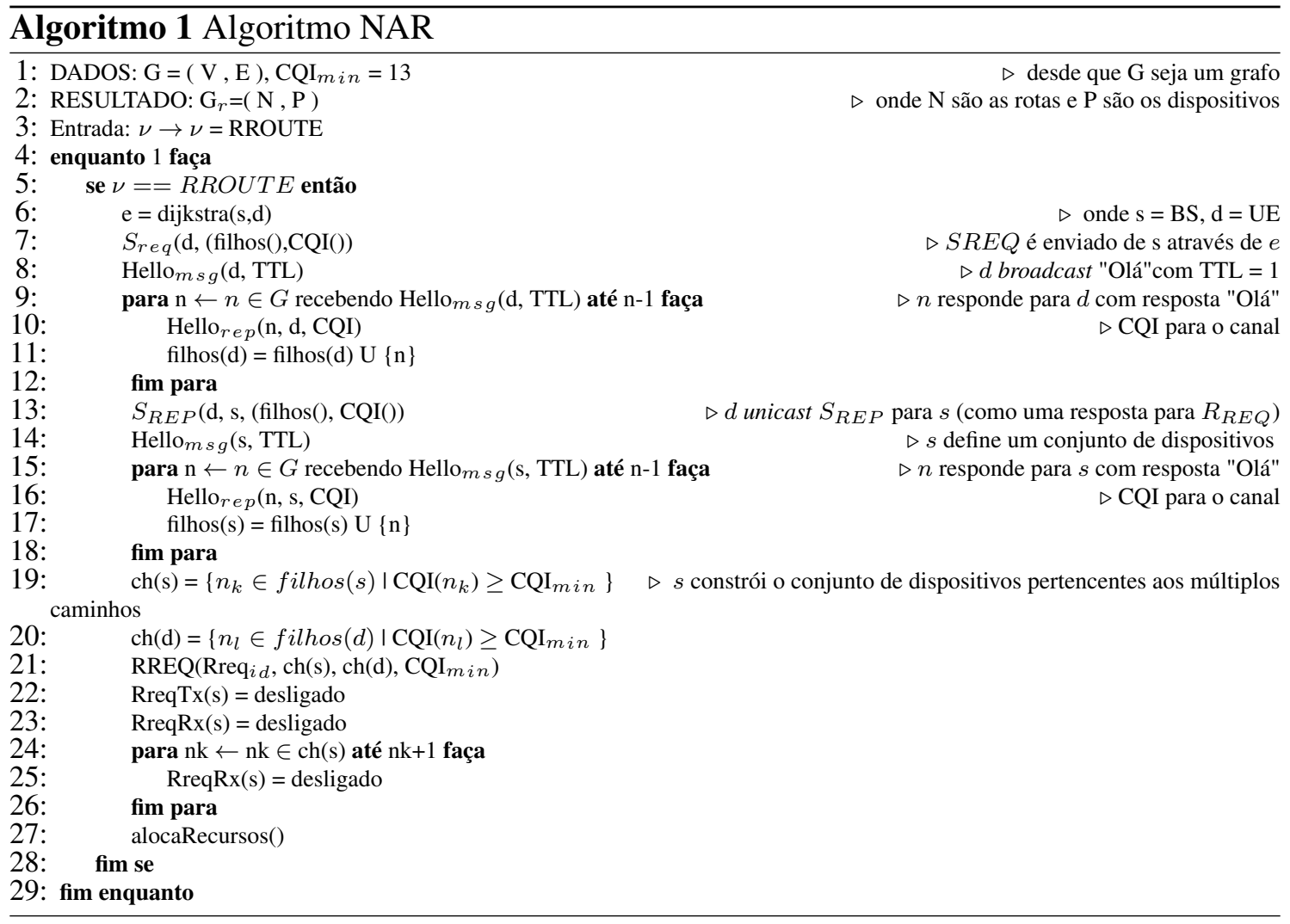

ção é feita para as eNBs para não ajustar o $R R E Q_{T X}$ para desativado até que ela envie um $R R E Q$ para todos os dispositivos escolhidos como membro de múltiplas rotas. Da mesma forma, o UE não ajusta o $R R E Q_{R X}$ para desativado, uma vez que não recebeu um $R R E Q$ de todos dispositivos escolhidos como membro de múltiplas rotas. Dessa forma, a eNB deve enviar um ( $R R E Q$ ) somente para os dispositivos escolhidos. Portanto, cada dispositivo precisa selecionar o melhor vizinho para qual o $R R E Q$ é encaminhado.

Uma vez que o UE recebe o $R R E Q$, ele responde com uma resposta de $R R E P$. $R R E P$ adiciona informações simples sobre o CQI do canal, permitindo a escolha de várias rotas. Após a definição das rotas, o procedimento de alocação de recursos, apresentado no Algoritmo 2 é executado em que cada pedido de rota (RROUTE) de um UE são definidos duas rotas com diferentes blocos de recursos, sendo uma rota para a comunicação D2D e a outra rota servindo como auxiliar em caso de falha. Para o encerramento da rota, a vida útil da rota foi definida em $60 \mathrm{~s}$. O algoritmo é considerado pró-ativo pois seleciona mais de uma rota para a comunicação D2D e reativo pois seleciona rotas somente após uma solicitação de rotas.

\section{Baseline}

Nosso algoritmo é comparado ao algoritmo Load Balancing Based Selective Ad hoc On-Demand Multipath Distance Vector (LBS-AOMDV) um aprimoramento do algoritmo AOMDV [Marina and Das 2001]. Ele é utilizado para definir múltiplas rotas entre as comunicações D2D nas Redes Heterogêneas [Baldini et al. 2014]. Desta forma, LBSAOMDV é um AOMDV seletivo. A escolha do algoritmo é devido ser um algoritmo bem conhecido na literatura para roteamento D2D, possuindo similaridades com a proposta apresentada. Uma das particularidades do LBS-AOMDV é oferecer informações sobre a 


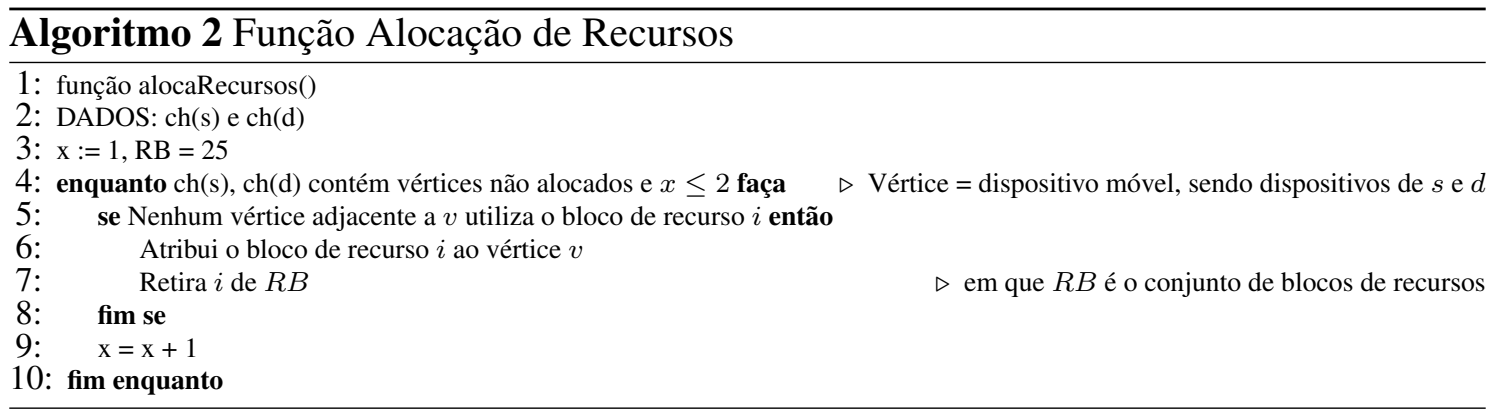

largura de banda disponível de cada rota nas múltiplas rotas. Além disso, reduz o tráfego de controle ao diminuir o número de nós que recebem os pedidos $R R E Q$, sendo viável uma vez que os $R R E Q$ selecionam os nós capazes de receberem os pacotes. O algoritmo encaminha $R R E Q$ da fonte para encontrar o destino, mas ao contrário do AOMDV, o nó remetente não inunda os pacotes através de toda a rede. Em vez disso, para cada transmissão, escolhe o melhor entre os seus filhos para ser o receptor $R R E Q$. A definição do melhor vizinho é descrito na Eq. 4:

$$
C h(p r)=\{c i / i \in N \text { edist }(p r, c i) \leq R\}
$$

Neste contexto, $(p r, c i)$ indica a distância entre o nó pai $p r$ e a criança $c i$, enquanto $R$ representa a cobertura de alcance dos nós na rede. O melhor dispositivo $c_{b}$ do nó origem $p r$ é aquele que pode fornecer a melhor quantidade de largura de banda (Best $B W)$. A quantidade de $B e s t B W$ é calculada dependendo da largura de banda disponível do conjunto de $p r$ dispositivos.

\section{Metodologia}

Para a validação do modelo e avaliação do algoritmo NAR, foram realizadas simulações no simulador SimuLTE [Virdis et al. 2014] analisando os efeitos da alocação de blocos de recursos na interferência da comunicação D2D e a comparação do algoritmo NAR com o baseline para avaliar a importância da participação das eNBs na definição de uma rota com menor interferência. Para realizar uma abordagem científica no planejamento experimental, foram definidos os parâmetros da simulação e a forma da coleta dos dados, para posteriormente realizar a análise dos dados, refutando ou corroborando o algoritmo proposto. A seguir uma descrição para cada fase realizada do planejamento experimental.

a) Reconhecimento e definição do problema: Garantir a confiabilidade na entrega das mensagens, minimizando a perda de mensagens sem inviabilizar o tempo da rede;

b) Escolha de fatores de interesse, níveis e intervalos: $\mathrm{Na}$ tabela 1 foram definidos os parâmetros utilizados nas simulações;

c) Seleção da variável de resposta: mensagens de dados enviadas, mensagem de dados perdidas, nível do indicador de qualidade do canal (CQI), número de fluxos D2D e energia consumida. Todas as variáveis foram analisadas para cada algoritmo avaliado, exceto o CQI que não está disponível para o algoritmo LBS-AOMDV.

O planejamento de experimentos foi baseado nas definições do planejamento préexperimental, sendo definidos os experimentos e as variáveis de respostas: a) Fatores de 


\begin{tabular}{|l|l|}
\hline Parâmetros & Valor \\
\hline Frequência da Portadora & $2 \mathrm{GHz}$ \\
Largura de Banda & $5 \mathrm{MHz}$ (25 RBs) \\
Modelo de Perda & ITU Urban Macro \\
Modelo de Desvanecimento & Jakes \\
Potência dispositivo móvel Tx & $26 \mathrm{~dB}$ \\
Ruído & $5 \mathrm{~dB}$ \\
Perda no Cabo & $2 \mathrm{~dB}$ \\
Tempo de Simulação & $3600 \mathrm{~s}$ \\
\hline
\end{tabular}

Tabela 1. Parâmetros da Simulação no SimuLTE.

interesse: mensagens de dados enviadas, mensagens de dados perdidas, nível do CQI, número de fluxos D2D e energia consumida; b) Hipótese Nula (H0): H0: $T 1=T 2=0$; c) $\mathbf{H 1}$ (unilateral / bilateral): $H 1: T i \neq 0 \rightarrow$ (Hipótese Alternativa) $\rightarrow$ para no mínimo um $i$; d) Nível de significância:: $\alpha=0.05$. Nesse contexto, $T 1$ e $T 2$ representam os algoritmos NAR e LBS-AOMDV e $\alpha$ a confiabilidade dos dados. As análises dos dados foram realizadas através da ferramenta R [Cano E.L. 2012].

Para a comparação entre os algoritmos, cada experimento foi realizado 33 vezes com duração de 3600 segundos para cada simulação conforme [Montgomery and Runger 2006], em que cada algoritmo e executado de acordo com os parâmetros descritos na tabela 1, em um cenário com uma estação base e 200 dispositivos móveis dispostos de forma estacionária e aleatória dentro de uma área de cobertura celular de $1000 \mathrm{~m}$ de raio. Os dispositivos móveis realizam comunicações celulares de forma aleatória e simultânea, sendo que o dispositivo móvel alocado para formar uma rota de comunicação D2D não pode realizar comunicação celular simultaneamente.

\section{Resultados}

Para a comparação do algoritmo NAR e o baseline, o objetivo foi analisar o funcionamento do algoritmo em relação as métricas: consumo de energia, mensagens enviadas e mensagens perdidas. Para todos os experimentos, a análise do CQI foi utilizada para determinar a distância entre os dispositivos móveis de forma a evitar perdas de pacotes.

\subsection{Qualidade do Canal}

Para o funcionamento adequado das comunicações D2D, a qualidade do canal (CQI) é um fator que interfere diretamente no funcionamento do algoritmo NAR. Na figura 2 é representado o CQI que determinada a qualidade do canal das comunicações para o algoritmo NAR em relação a distância (metros).

Quanto maior for o valor do CQI, melhor é a qualidade do canal. Na simulação, foram determinados os 15 valores de CQI apresentados no release 8 do LTE-A. Esse resultado indica que uma distância $(R)<80 \mathrm{~m}$ pode garantir uma boa qualidade do canal.

\subsection{Consumo de Energia}

Para a avaliação do consumo energético nos dispositivos móveis, foram definidos alguns parâmetros para avaliar o consumo de energia na comunicação D2D, como por exemplo, a potência consumida para transmissão de mensagens de dados e roteamento. A potência consumida para recepção de mensagens e mensagens de controle não foram contabilizados nas simulações. A tabela 2 apresenta algumas informações sobre a média de mensagens enviadas e a energia consumida pelos dispositivos móveis. 


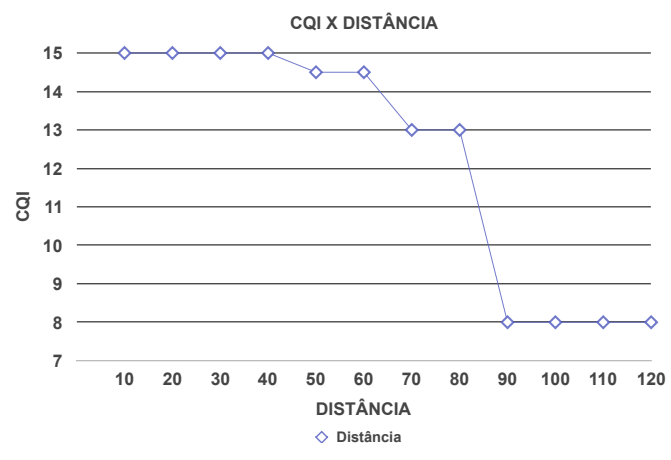

Figura 2. Qualidade do canal - CQI

\begin{tabular}{|l|l|l|}
\hline Descrição & NAR & LBS \\
\hline Energia Inicial $(\mathrm{J})$ & 10000 & 10000 \\
Mensagens Dados & 17496 & 11563 \\
Mensagens Roteamento & 1603 & 2207 \\
Total de Pacotes & 19099 & 13770 \\
Potência Consumida para transmissão $(\mathrm{mW})$ & 660 & 660 \\
Tempo de Simulação & $3600 \mathrm{~s}$ & $3600 \mathrm{~s}$ \\
Total de Energia Consumida (Média) & 5250 & 6225 \\
\hline
\end{tabular}

Tabela 2. Energia Consumida

Observando-se o boxplot da figura 3(a) referente as amostras de energia consumida e analisando modelo linear balizado pelo teste $\mathrm{f}$, em que o valor de $p$ para a ANOVA foi de $2 e-16$, observamos uma diferença significativa entre os algoritmos, rejeitando a hipótese nula de médias iguais entre as amostras. Através do teste de Fisher com correção de Bonferroni [Montgomery and Runger 2006] é possível fazer comparações entre os pares das médias e verificar através da figura 3(b) diferenças significativas entre as amostras.

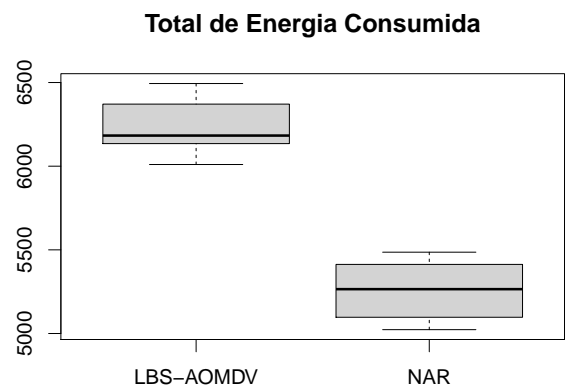

(a) BoxPlot - Energia Consumida

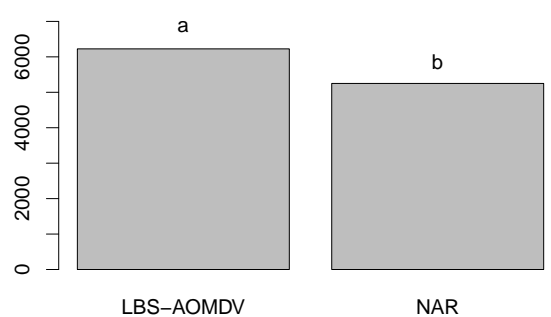

(b) Teste de Fisher.

Figura 3. Energia Consumida

\subsection{Mensagens Perdidas}

Observando-se o boxplot da figura 4(a) referente as amostras de mensagens perdidas e analisando modelo linear balizado pelo teste $\mathrm{f}$, em que o valor de $p$ para a ANOVA foi de 
$2 e-16$, observamos uma diferença significativa entre os algoritmos, rejeitando a hipótese nula de médias iguais entre as amostras. Através do teste de Fisher com correção de Bonferroni [Montgomery and Runger 2006] é possível fazer comparações entre os pares das médias e verificar através da figura 4(b) diferenças significativas entre as amostras.

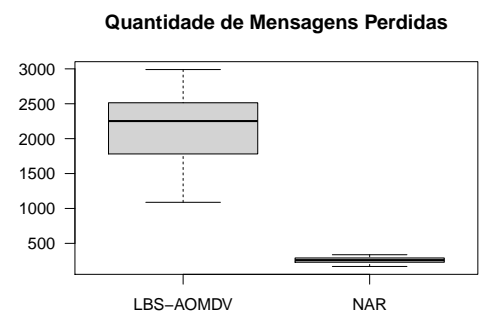

(a) BoxPlot - Mensagens Perdidas

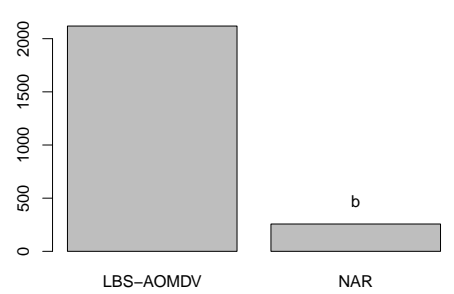

(b) Teste de Fisher.

Figura 4. Mensagens Perdidas

\subsection{Mensagens Enviadas}

Observando-se o boxplot da figura 5(a) referente as amostras de mensagens enviadas e analisando modelo linear balizado pelo teste $\mathrm{f}$, em que o valor de $p$ para a ANOVA foi de $2 e-16$, observamos uma diferença significativa entre os algoritmos, rejeitando a hipótese nula de médias iguais entre as amostras. Através do teste de Fisher com correção de Bonferroni [Montgomery and Runger 2006] é possível fazer comparações entre os pares das médias e verificar através da figura 5(b) diferenças significativas entre as amostras.

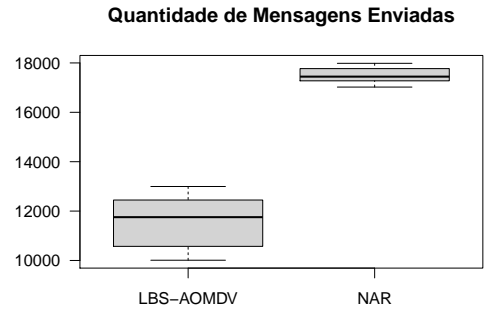

(a) BoxPlot - Mensagens Perdidas

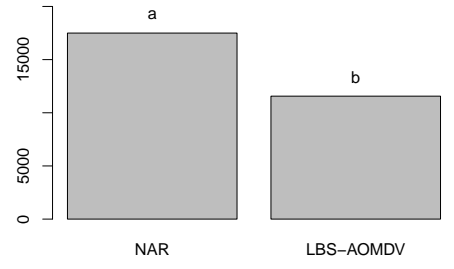

(b) Teste de Fisher.

Figura 5. Mensagens Enviadas

\subsection{Alocação de Blocos de Recursos}

Para avaliar o impacto da interferência na alocação de recursos, simulamos a proposta da alocação de blocos de recursos em um cenário composto por uma eNB e uma variância de 100 a 1000 UEs distantes entre eles em até $70 \mathrm{~m}$, sendo distribuídos aleatoriamente até $2000 \mathrm{~m}$ de distância da eNB. Cada UE envia pacotes de 100 bytes a cada $20 \mathrm{~ms}$, resultando em um tráfego de taxa de bits constante (CBR) de (aproximadamente) $1757 \mathrm{kbps}$, sendo o período de coleta do CQI definido como $10 \mathrm{~ms}$. 


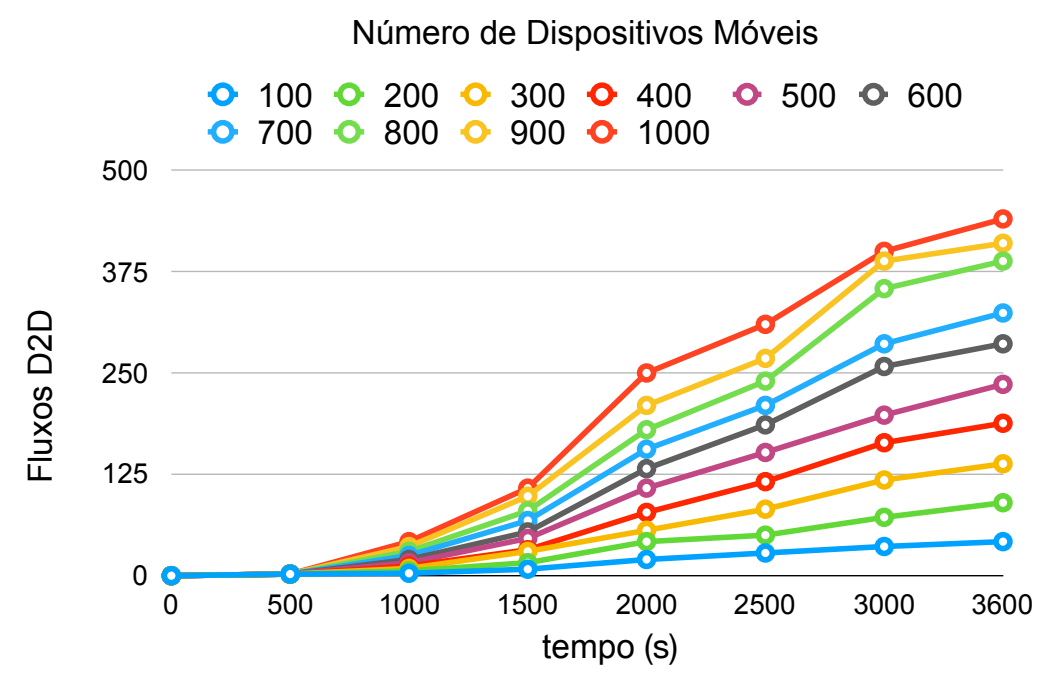

Figura 6. Número de Fluxos D2D.

A figura 6 demonstra o número de fluxos durante um determinado período de tempo. Esses valores são obtidos executando o sistema em uma CPU Intel (R) Core i5 3,0 GHz com 8 GB RAM, sistema operacional Linux Ubuntu 16.04 e OMNeT ++ versão 4.6. SimuLTE versão 2.4. Como esperado, a interferência computacional requer tempo adicional de CPU, que depende do número de fluxos de rede. Sendo a avaliação da relação sinal-ruído (SINR) recebida na presença de interferência requer ciclos através de todos os dispositivos móveis para verificar se eles estão transmitindo no mesmo bloco de recursos.

\section{Conclusão}

Esse trabalho apresenta o algoritmo NAR para comunicação D2D nas redes celulares assumindo que o roteamento seja definido pela estação base. NAR depende da qualidade do canal e da alocação de blocos de recursos baseado no problema de coloração de grafos para definir as rotas, a fim de reduzir o risco de interferência entre as comunicações adjacentes, ao mesmo tempo em que melhora o uso do espectro celular.

Os experimentos foram realizados no SimuLTE em que o NAR foi comparado com o baseline LBS-AOMDV. Os resultados demonstram que o NAR tem um ganho energético de aproximadamente $16 \%$ comparado ao LBS-AOMDV, enquanto envia aproximadamente $51 \%$ a mais de mensagens em relação ao LBS-AOMDV.

Através dos experimentos realizados na comparação do algoritmo NAR em relação ao algoritmo LBS-AOMDV, foi possível verificar que a abordagem proposta para o roteamento, tendo como premissa uma participação efetiva da estação base na determinação da rota, na escolha da rota baseado no CQI do canal e a alocação de blocos de recursos para cada rota, permitiu definir uma rota com menor interferência e melhor entrega das mensagens, contribuindo para uma diminuição no consumo energético dos dispositivos móveis, refutando a Hipótese Nula com um nível de significância de 95\%. Como próximos passos, busca-se a adaptação do NAR para um cenário com UEs móveis. 


\section{Agradecimentos}

Esse trabalho foi realizado com apoio financeiro do CNPq, CAPES, FAPEMIG e DTECH/UFSJ.

\section{Referências}

Agiwal, M., Roy, A., and Saxena, N. (2016). Next generation 5G wireless networks: A comprehensive survey. IEEE Communications Surveys and Tutorials, 18(3):16171655 .

Asadi, A., Wang, Q., and Mancuso, V. (2014). A survey on device-to-device communication in cellular networks. IEEE Communications Surveys and Tutorials, 16(4):18011819.

Baldini, G., Karanasios, S., Allen, D., and Vergari, F. (2014). Survey of Wireless Communication Technologies for Public Safety. Communications Surveys Tutorials, IEEE, 16(2):619-641.

Bastos, A. V., S. C. M. and Silva, D. C. J. (2017). NAR : Algoritmo de Roteamento Assistido para Comunicação D2D em Redes Celulares de 5G. IX SBCUP (Simpósio Brasileiro de Computação Ubíqua e Pervasiva).

Bastos, A. V., S. C. M. and Silva, D. C. J. (2018). Assisted Routing Algorithm for D2D Communication in 5G Wireless Networks. 2018 Wireless Days (WD) (WD'18).

Cano E.L., Moguerza J.M., R. A. (2012). Design of Experiments with R, volume 36. Springer.

Felice, D. M., B. L. and Bononi, L. (2016). The Emergency Direct Mobile App: Safety Message Dissemination over a Multi-Group Network of Smartphones using Wi-Fi Direct. 14th ACM International Symposium on Mobility Management and Wireless Access, (November):99-106.

Jiang, J., Zhu, Y., Li, B., and Li, B. (2015). Rally: Device-to-device content sharing in LTE networks as a game. Proceedings - IEEE 12th International Conference on Mobile Ad Hoc and Sensor Systems, MASS 2015, pages 10-18.

Jingyi, Z., Xi, L., and Quansheng, X. (2015). Multi-hop routing for energy-efficiency enhancement in relay-assisted device-to-device communication. Journal of China Universities of Posts and Telecommunications.

Laha, A., Cao, X., Shen, W., Tian, X., and Cheng, Y. (2015). An energy efficient routing protocol for device-to-device based multihop smartphone networks. IEEE International Conference on Communications, 2015-Septe:5448-5453.

Lee, J., G. J. B. J. S. and Chung, Y. M. (2013). A resource allocation scheme for improving user fairness in device-to-device communication based on cellular networks. ICUIMC 13 - Proceedings of the 7th International Conference on Ubiquitous Information Management and Communication, (112).

Lin, X., Andrews, J. G., Ghosh, A., and Ratasuk, R. (2014). An overview of 3GPP device-to-device proximity services. IEEE Communications Magazine, 52(4):40-48. 
Marina, M. K. and Das, S. R. (2001). On Demand Multipath Distance Vector Routing in Ad Hoc Networks. Proc. IEEE International Conference on Network Protocols (ICNP'01), pages 14-23.

Mehrotra, A. and Trick, M. A. (1995). A Column Generation Approach For Graph Coloring. INFORMS Journal on Computing, 8:344-354.

Melki, L., Najeh, S., and Besbes, H. (2016). Interference Management Scheme for Network-Assisted Multi-Hop D2D Communications. IEEE 27th Annual International Symposium on Personal, Indoor and Mobile Radio Communications (PIMRC), pages $1-5$.

Montgomery, D. C. and Runger, G. C. (2006). Applied Statistics and Probability for Engineers, volume 4th. John Wiley and Sons Wiley.

Nardini, G. and Stea, b. G. (2016). Resource allocation for network-controlled deviceto-device communications in LTE-Advanced. Springer Wireless Networks journal, (January):1-24.

Nunes, O. I., M. V. S. O. P. and Loureiro, F. A. A. (2016). GROUPS-NET: Roteamento Ciente de Encontros de Grupos em Redes Móveis D2D. XXXIV Simpósio Brasileiro de Redes de Computadores e Sistemas Distribuídos - SBRC 2016.

Sedidi, R. and Kumar, A. (2016). Key exchange protocols for secure Device-to-Device (D2D) communication in 5G. IFIP Wireless Days, 2016-April:1-6.

Sharma, A., T. A. and Roberts, N. (2015). Efficient Load Balancing using D2D Communication and Biasing in LTE-Advance Het-Nets. ICCCT 15 - Proceedings of the Sixth International Conference on Computer and Communication Technology, pages 456-460.

Shaw, J. A. (2012). Radiometry and the Friis transmission equation. American Journal of Physics.

Tata, C. and Kadoch, M. (2014). Multipath routing algorithm for device-to-device communications for public safety over LTE Heterogeneous Networks. 2014 1st International Conference on Information and Communication Technologies for Disaster Management, ICT-DM 2014.

Virdis, A., Stea, G., and Nardini, G. (2014). SimuLTE - A Modular System-level Simulator for LTE / LTE-A Networks based on OMNeT ++. Proceedings of SimulTech, pages $28-30$.

Wang, S., Guo, W., Zhou, Z., Wu, Y., and Chu, X. (2015). Theoretical Framework for Multi-hop D2D Communications with Shortest Path Routing. IEEE Communications Letters, 19(11):1-4.

Yuan, H., Guo, W., and Wang, S. (2014). Emergency route selection for D2D cellular communications during an urban terrorist attack. 2014 IEEE International Conference on Communications Workshops, ICC 2014, pages 237-242.

Zhu, Y., Jiang, J., Li, B., and Li, B. (2015). Rado: A Randomized Auction Approach for Data Offloading via D2D Communication. IEEE 12th International Conference on Mobile Ad Hoc and Sensor Systems, MASS 2015, pages 1-9. 